\title{
Broadband notch filter design for millimeter-wave plasma diagnostics
}

Furtula, Vedran; Michelsen, Poul; Leipold, Frank; Salewski, Mirko; Korsholm, Søren Bang; Meo, Fernando; Nielsen, Stefan Kragh; Stejner Pedersen, Morten; Moseev, Dmitry; Johansen, Tom Keinicke

Published in:

Review of Scientific Instruments

Link to article, DOI:

$10.1063 / 1.3478881$

Publication date:

2010

Link back to DTU Orbit

Citation (APA):

Furtula, V., Michelsen, P., Leipold, F., Salewski, M., Korsholm, S. B., Meo, F., Nielsen, S. K., Stejner Pedersen, M., Moseev, D., \& Johansen, T. K. (2010). Broadband notch filter design for millimeter-wave plasma diagnostics. Review of Scientific Instruments, 81(10), 10D913 (5 pages). https://doi.org/10.1063/1.3478881

\section{General rights}

Copyright and moral rights for the publications made accessible in the public portal are retained by the authors and/or other copyright owners and it is a condition of accessing publications that users recognise and abide by the legal requirements associated with these rights.

- Users may download and print one copy of any publication from the public portal for the purpose of private study or research.

- You may not further distribute the material or use it for any profit-making activity or commercial gain

- You may freely distribute the URL identifying the publication in the public portal 


\title{
Broadband Notch Filter Design for mm-Wave Plasma Diagnostics
}

\author{
V. Furtula, P. K. Michelsen, F. Leipold, M. Salewski, S. B. Korsholm, F. Meo, S. K. Nielsen, M. Stejner, and D. Moseev \\ Association Euratom - Risø National Laboratory for Sustainable Energy, \\ Technical University of Denmark, DK-4000 Roskilde \\ T. Johansen \\ DTU Elektro, Technical University of Denmark, DK-2800 Lyngby
}

(Dated: September 3, 2010)

\begin{abstract}
Notch filters are integrated in plasma diagnostic systems to protect mm-wave receivers from intensive stray radiation. Here we present a design of a notch filter with a center frequency of $140 \mathrm{GHz}$, a rejection bandwidth of $\sim 900 \mathrm{MHz}$, and a typical insertion loss below $2 \mathrm{~dB}$ in the passband of $\pm 9 \mathrm{GHz}$. The design is based on a fundamental rectangular waveguide with 8 cylindrical cavities coupled by T-junction apertures formed as thin slits. Parameters that affect the notch performance such as physical lengths and conductor materials are discussed. The excited resonance mode in the cylindrical cavities is the fundamental $\mathrm{TE}_{11}$. The performance of the constructed filter is measured using a vector network analyzer monitoring a total bandwidth of $30 \mathrm{GHz}$. We compare the measurements with numerical simulations.
\end{abstract}

\section{INTRODUCTION}

Millimeter ( $\mathrm{mm})$ wave notch filters are useful in different mm-wave plasma diagnostics such as electron cyclotron emission (ECE), reflectometry, and collective Thomson scattering (CTS) as they protect delicate microwave components from gyrotron stray radiation [14]. Many of today's magnetically confined plasma devices are equipped with high power gyrotrons in the megawatt range. ASDEX Upgrade (AUG) is equipped with $140 \mathrm{GHz}$ gyrotrons and additional new dual frequency gyrotrons capable of operating at either 105 or $140 \mathrm{GHz}[5,6]$. Purposes of the gyrotrons range from heating, current drive, mitigating of tearing modes $[5,6]$, and stabilizing sawteeth. The different frequencies enable more flexibility in operating at different scenarios. The $105 \mathrm{GHz}$ operation is also used as the scattering probe for the CTS diagnostic [1-4]. Diagnostics viewing the plasma within this frequency range, for example ECE or CTS, need to be protected from stray radiation coming from the gyrotrons. For a single gyrotron, the notch width needs to cover the frequency temporal evolution due to chirps during ramp-up and frequency drifts during discharge operation due to the gyrotron cavity thermal expansion. Hence usually $150-200 \mathrm{MHz}$ is needed. When several gyrotrons are used, a wider notch width is required since frequency offsets between the gyrotrons are usually in the sub $\mathrm{GHz}$ range for the same gyrotron mode [6]. The typical passband width of D-band notch filters is few $\mathrm{GHz}[7,8]$ with notch center frequency $f_{0}$ located in the range $90-140 \mathrm{GHz}$. In this paper we show how to broad out the passband coverage significantly and still maintain the insertion loss $(<2 \mathrm{~dB})$, making the notch filters suitable for ECE applications.

To extract satisfactory diagnostic data, the bandwidth of the passband on each side of the notch has to be adequate where at the same time insertion loss (IL) of the notch filter should be as small as possible and independent of the frequency. In addition, the larger the pass- band coverage, the more likely the same notch filter can be used for several diagnostics reducing the overall system cost. Due to their robustness to high radiative and neutron flux environment, mm-wave based diagnostics will play a key role in future burning plasma devices [9]. Hence high performing notch filters will play an important role in a strongly radiative environment.

A major part in this paper will focus on sensitivity analysis on various filter parameters. In section II we discuss background and theory on rectangular and circular waveguides. In section III we discuss numerical methods and simulation results while in section IV we focus on experimental results.

\section{SLIT-COUPLED T-JUNCTION FOR WAVEGUIDES}

H.A. Bethe developed a perturbation technique to calculate the power transmission through and reflection by small irises (slits) in standard waveguides [10]. Bethe's approach is based on the following two steps. The first step is to calculate the electric dipole moment, $\overline{\mathbf{p}}$, and the magnetic dipole moment, $\overline{\mathbf{m}}$, induced in the slit by the exciting fields. The next step is to calculate the power radiated due to the electric and magnetic dipole moments. The emanating total fields are calculated from electric and magnetic equivalent sources, a technique also used for aperture antennas. The equivalent sources take the form of short dipoles when the radiating structure/gap/hole is sufficiently small compared with the vacuum wavelength $\lambda_{0}$.

Waveguide notch filters can be designed using several different constellations out of which we consider one. The main waveguide has standard dimensions, and the resonators have circular cross sections. The lengths, depths, and widths of the coupling slits and the cavity resonator lengths are the degrees of freedom. The optimum insertion loss characteristic is found from a trade-off in these 
parameters.

The geometrical layout behind the $140 \mathrm{GHz}$ notch filter is adapted from the resonator-coupling figure presented by Rizzi [11, p.434] and is shown in fig. 1 . This notch filter construction is composed of a symmetrical rightangle T-junction of two waveguides which are coupled by a narrow slit in a wall that is electrically thin, i.e. the slit width is much smaller than $\lambda_{0}=c / f_{0}=2.1 \mathrm{~mm}$. The slit widths are restricted to a minimum value for a certain machining technology, usually $>0.1 \mathrm{~mm}$, which is much less than $2.1 \mathrm{~mm}$ as required. Later we show that the slit width has large impact on $3 \mathrm{~dB}$ rejection bandwidth.

The resonating cavities at the end of each T-junction can be designed in several manners. The simplest way is to use cylindrical cavities due to the simple tuning screw mechanism. The resonators with a diameter of $D=1.31 \mathrm{~mm}$ are placed equidistant along the main waveguide. The electric field component of the fundamental $\mathrm{TE}_{10}$ rectangular guide mode is coupled to the electric field component of the $\mathrm{TE}_{11 p}$ circular cavity mode with a thin slit $[11, \mathrm{p} .431]$. The subscript $p$ is a number of $\lambda_{g} / 2$ multiples in the resonator. $\lambda_{g}$ is the guide wavelength and $l$ is the cavity length given as

$$
\lambda_{g}=\frac{\lambda_{0}}{\sqrt{1-\left(\frac{\lambda_{0}}{\lambda_{c}}\right)^{2}}}, \quad \text { and } \quad l=p \frac{\lambda_{g}}{2} .
$$

$\lambda_{c}$ is the cut-off wavelength for the specific geometry [11, p.460]. For the circular waveguide, $\lambda_{c, n m}=\pi D / p_{n m}$ where $p_{n m}$ correspond to the m'th root of the derivative of first order Bessel function. For the $\mathrm{TE}_{11 p}$ mode we have $\lambda_{c, 11}=1.31 \pi / 1.841=2.24 \mathrm{~mm}$ and $\lambda_{g}=$ $7.4 \mathrm{~mm}$. This gives the possible cavity lengths $l=$ $\{3.7,7.4 \ldots\} \mathrm{mm}$. The distance between cavities in fig. 2 is determined as an odd number of quarter wavelengths

$$
\Delta l=\frac{\lambda_{g}}{4}(1+2 N),
$$

where $N=\{0,1,2 \ldots\}$ and $\lambda_{g}$ is the guide wavelength for the main rectangular guide. We first find $\lambda_{c}=2 a=$ $3.30 \mathrm{~mm}$, where $a=1.651 \mathrm{~mm}$ is the broad side of the main guide and then using eq. (1) we find $\lambda_{g}=2.8 \mathrm{~mm}$. Due to geometrical limitations we set $N=1$, that gives $\Delta l=2.1 \mathrm{~mm}$ which is the value we use in the design phase. A planar cut of the simulated notch filter and photo of the realized notch filter are shown in fig. 2 .

\section{NUMERICAL METHODS AND RESULTS}

Numerical simulations are used to optimize the coupling slit dimensions mentioned in Sec. II. Maxwell's equations with boundary conditions describe the electromagnetic field distribution inside a waveguide, the coupling slits, and resonator cavities [11, pp.24-28]. CST [12] is a time domain solver based on the finite element

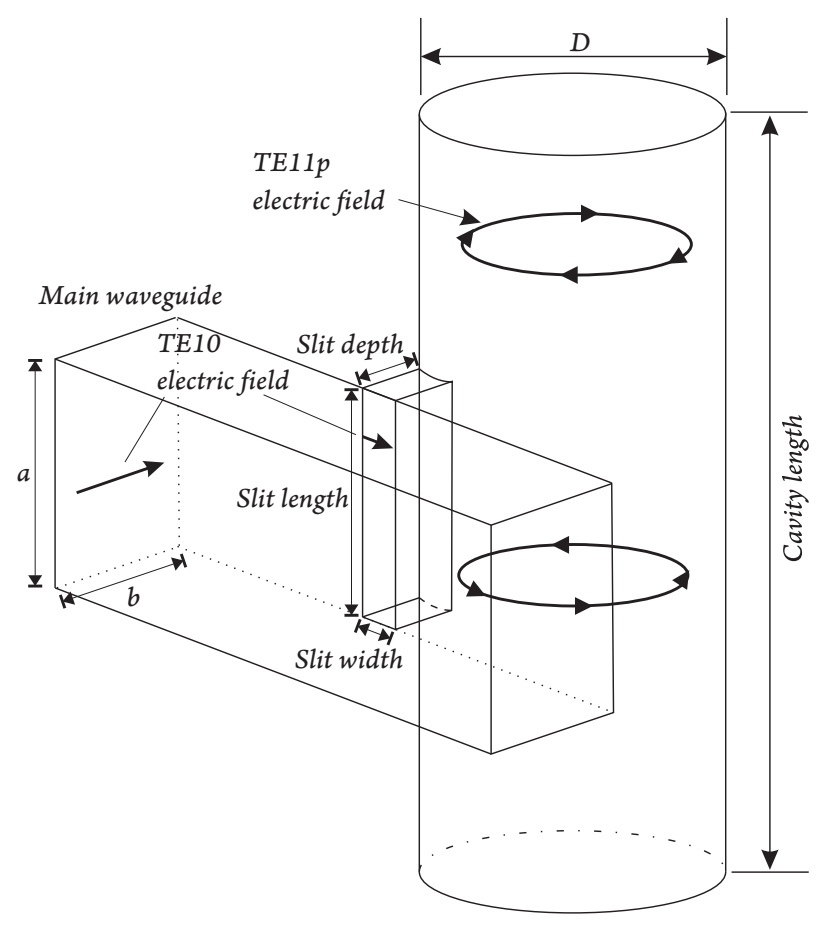

FIG. 1. T-junction aperture coupling using a thin slit and vertical cavity scheme. The fixed dimensions are $a=1.651 \mathrm{~mm}$, $b=0.826 \mathrm{~mm}$ and $D=1.31 \mathrm{~mm}$.

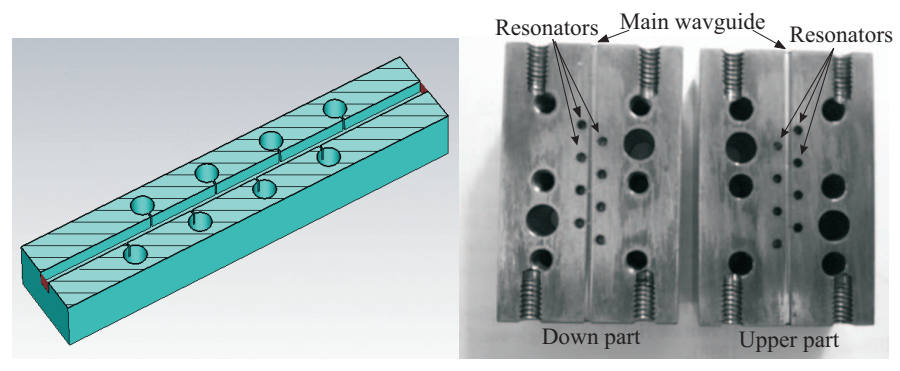

FIG. 2. 3-D horizontal cut and photography of the $140 \mathrm{GHz}$ notch filter with eight cavities.

method used to calculate the fields existing inside the structure. The output from the solver is the frequency dependent scattering parameter (S-parameter) $S_{21}$, better known as insertion loss or transmission loss.

We compute the results on various meshes with increasingly finer resolution to test the convergence. Figure 3 shows the notch frequency $f_{0}$, the $3 \mathrm{~dB}$ bandwidth, and the shape factor as a function of mesh size, here calculated as lines per wavelength $\left(L / \lambda_{0}\right)$ where $L / \lambda_{0}=22$ corresponds to 2.6 million meshcells. The center frequency $f_{0}$ shifts down $500 \mathrm{MHz}$ when the grid resolution is increased from 8 to 22 lines per wavelength. The $3 \mathrm{~dB}$ bandwidth oscillates between 750 and $900 \mathrm{MHz}$ for low values of lines per wavelength (8 to 18). At 20 lines per wavelength, the $3 \mathrm{~dB}$ bandwidth has settled to $\sim 840 \mathrm{MHz}$. The shape factor is a ratio of the bandwidths at two different stopband levels: $\mathrm{IL}=3 \mathrm{~dB}$ (half power) 

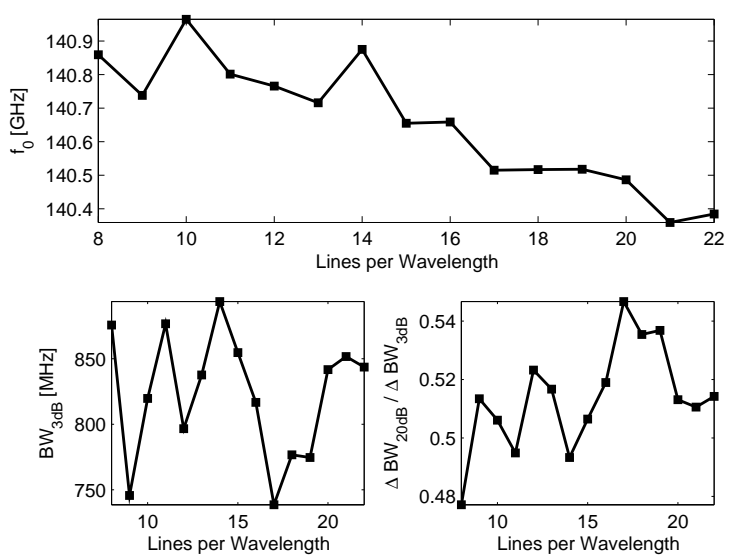

FIG. 3. Center frequency (upper), $3 \mathrm{~dB}$ bandwidth (lowerleft), and shape factor (lower-right) are plotted versus lines per wavelength $\left(\mathrm{L} / \lambda_{0}\right)$.

and $\mathrm{IL}=20 \mathrm{~dB}$ ( $1 \%$ power transmission), i.e. shape factor $=\mathrm{BW}_{20 d B} / \mathrm{BW}_{3 d B}$ and is a number between 0 and 1 . A desired scenario is that $3 \mathrm{~dB}$ points and $20 \mathrm{~dB}$ points have equal bandwidths giving ideal selectivity in form of two vertical lines when $S_{21}$ is plotted versus frequency, i.e. shape factor $=1$. In fig. 3 the shape factor changes only slightly from 0.48 up to 0.52 , so the computed filter selectivity is independent of the grid. Due to $3 \mathrm{~dB}$ bandwidth uncertainties in fig. 3 (lower-left), we choose the highest applicable number of lines per wavelength in order to achieve reliable S-parameter values. The simulations shown in this paper will be that of 20 lines per wavelength as a compromise between the calculated filter parameter accuracy and simulation time.

All narrowband mm-wave filters, i.e. $\mathrm{f}_{0} / \mathrm{BW}_{3 d B} \gg 1$, are sensitive to mechanical imperfections and therefore should be tunable. In our case, the only adjustable parameter is the length of the cylindrical cavities. This filter has eight cavities and their respective lengths are identical for each simulation run. Individual cavity tuning is time consuming. In the following sensitivity analysis of the geometrical parameters will be presented. Some of the data sets are aligned so well that linear least squares fit is applied. Otherwise, the highest absolute gradient among the points is calculated, i.e. the highest sensitivity.

Table I shows the sensitivity overview of the filter design parameters to the geometrical changes. The center frequency in fig. 4 (upper) varies linearly as a function of cavity length with a slope of $-1.43 \mathrm{GHz} / \mathrm{mm}$. The cavity length is $5.8 \mathrm{~mm}$, for all 8 cavities, when centered at $140 \mathrm{GHz}$. The $3 \mathrm{~dB}$ bandwidth, $\mathrm{BW}_{3 d B}$, in fig. 4 (lower-left) is also linear in its response and changes with $-130 \mathrm{MHz} / \mathrm{mm}$ which is an acceptable value. Ideally, we prefer no change in the filter bandwidth (low sensitivity) when the cavities are being tuned. The worst case shape factor in fig. 4 (lower-right) has a relatively small
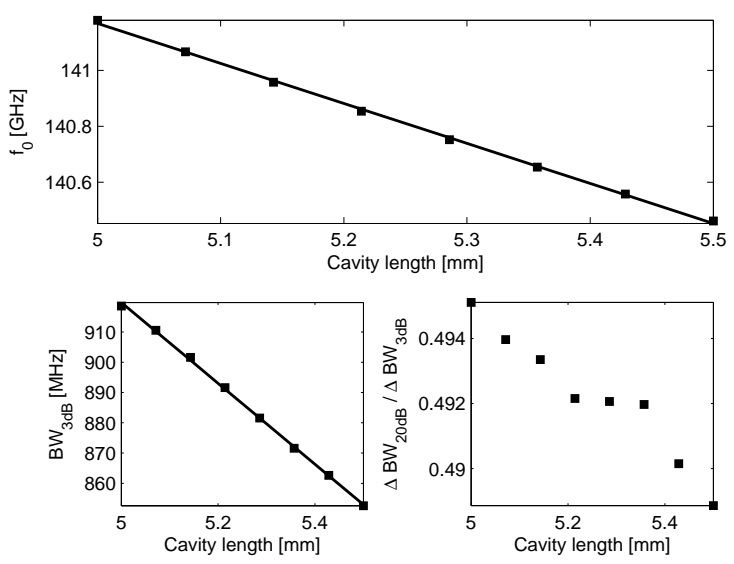

FIG. 4. Sensitivity of the center frequency $f_{0}$, the $3 \mathrm{~dB}$ bandwidth, and the shape factor to changes in the cavity length, see fig. 1. Symbols: simulations; line: least squares fit.
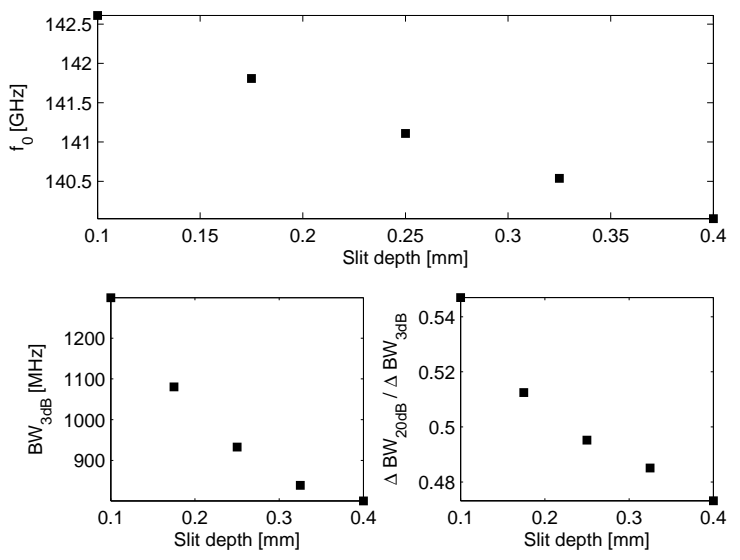

FIG. 5. Sensitivity of the center frequency $f_{0}$, the $3 \mathrm{~dB}$ bandwidth, and the shape factor to changes in the slit depth, see fig. 1 .

change by -0.026 per $\mathrm{mm}$. The $\mathrm{BW}_{3 d B}$ bandwidth is linear for small perturbations in cavity length with a slope of $130 \mathrm{MHz}$ per mm. It is possible to improve the selectivity of the filter by tuning cavity lengths individually to slightly different frequencies. This process has not been investigated. The main purpose of cavity tuning is to adjust the center frequency $f_{0}$ to the point of resonance.

The slit depth (fig. 5) is a nontunable parameter, so choosing this value carefully is important. The worst case $f_{0}$ gradient is $-10.71 \mathrm{GHz} / \mathrm{mm}$, which according to table I is the most sensitive parameter. The $3 \mathrm{~dB}$ bandwidth slope is $-2.92 \mathrm{GHz} / \mathrm{mm}$. The shape factor slope is low -0.459 per $\mathrm{mm}$, which is considered good. Choosing a slit depth of $0.28 \mathrm{~mm}$ results in a $3 \mathrm{~dB}$ bandwidth of $900 \mathrm{MHz}$ as required.

Figure 6 shows the effect of changing the slit width and is found to be a relatively sensitive parameter in 

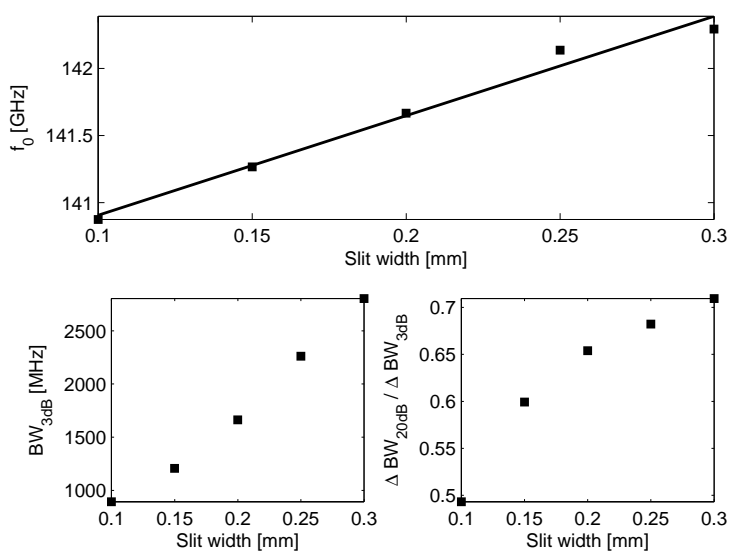

FIG. 6. Sensitivity of the center frequency $f_{0}$, the $3 \mathrm{~dB}$ bandwidth, and the shape factor to changes in the slit width, see fig. 1. Symbols: simulations; line: least squares fit.
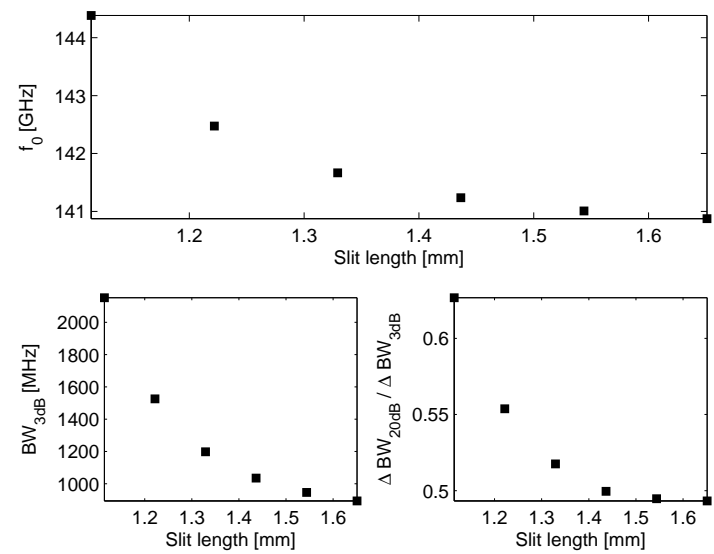

FIG. 7. Sensitivity of the center frequency $f_{0}$, the $3 \mathrm{~dB}$ bandwidth, and the shape factor to changes in the slit length, see fig. 1 .

all three categories: center frequency $f_{0}$ has a slope of $7.41 \mathrm{GHz} / \mathrm{mm}$, and $3 \mathrm{~dB}$ bandwidth has local gradients up to $11.96 \mathrm{GHz} / \mathrm{mm}$ in the range of interest. However, the shape factor grows with slit width. The slit width is chosen to be $0.1 \mathrm{~mm}$, which is the the lowest possible value due to machining limitations.

The center frequency and $3 \mathrm{~dB}$ bandwidth are very sensitive to the slit length as shown in fig. 7. From fig. 7 (lower-left) we choose the slit height to be equal to side $a$ of the main waveguide, $1.651 \mathrm{~mm}$, otherwise the $3 \mathrm{~dB}$ bandwidth will become too large.

The material choice is an important factor when minimizing the overall passband transmission loss, especially at the points around the rejection band. A clear difference in $3 \mathrm{~dB}$ bandwidth and shape factor from good to very good conductor is shown in fig. 8. As presented in fig. 8 (lower-left), the $3 \mathrm{~dB}$ bandwidth drops by $30 \%$
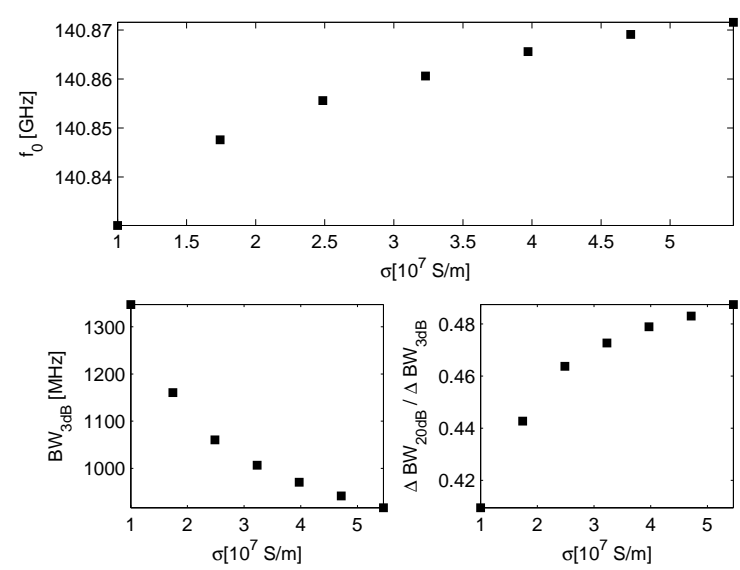

FIG. 8. Center frequency (upper), $3 \mathrm{~dB}$ bandwidth (lowerleft), and shape factor (lower-right) are plotted versus conductivity corresponding to good conductors.

\begin{tabular}{|c|c|c|c|}
\hline & $\begin{array}{c}f_{0} \\
{[\mathrm{GHz} / \mathrm{mm}]}\end{array}$ & $\begin{array}{c}3 \mathrm{~dB} \text { bandwidth } \\
{[\mathrm{GHz} / \mathrm{mm}]}\end{array}$ & $\begin{array}{c}\text { Shape factor } \\
{\left[\mathrm{mm}^{-1}\right]}\end{array}$ \\
\hline Cavity length & $\mathbf{- 1 . 4 3}$ & $\mathbf{- 0 . 1 3}$ & -0.026 \\
\hline Slit length & -17.83 & -5.85 & -0.681 \\
\hline Slit width & $\mathbf{7 . 4 1}$ & 11.96 & 2.121 \\
\hline Slit depth & -10.71 & -2.92 & -0.459 \\
\hline
\end{tabular}

TABLE I. Sensitivity analysis: impact on key characterization parameters by varying the design lengths. Bold numbers are found by least squares fit.

when changing conductivity from $10^{7} \mathrm{~S} / \mathrm{m}$ to $6 \cdot 10^{7} \mathrm{~S} / \mathrm{m}$. It is in agreement with microwave theory that the filter quality factor $Q \approx f_{0} / B W_{3 d B}$ improves with conductivity. The shape factor increases accordingly. Commonly either copper- $\left(\sigma=5.96 \cdot 10^{7} \mathrm{~S} / \mathrm{m}\right)$ or silver-coating $\left(\sigma=6.3 \cdot 10^{7} \mathrm{~S} / \mathrm{m}\right)$ are used as a construction material for frequencies above $110 \mathrm{GHz}$. For frequencies between 30 and $110 \mathrm{GHz}$ it is recommended to use gold $\left(\sigma=4.52 \cdot 10^{7} \mathrm{~S} / \mathrm{m}\right)$ or copper leaving silver-coating as an option.

\section{EXPERIMENTAL RESULTS AND DISCUSSION}

Eight resonator cavities are found to be a good compromise between filter performance and design complexity (see fig. 2). The distance between resonators $\Delta l$ in eq. (2) was optimized to $\Delta l \simeq \lambda_{g} / 4 \simeq 2.1 \mathrm{~mm}$ which fulfilled the requirement that the cylindrical resonators should be placed closely together to minimize insertion loss without any geometrical conflict. The eight cavities were tuned one at a time until their respective resonances were positioned at the center frequency of $140 \mathrm{GHz}$.

Based on the simulation findings, a notch filter with the following coupling geometry has been designed and built from copper: slit width of $0.1 \mathrm{~mm}$, slit depth of $0.28 \mathrm{~mm}$, and slit length of $1.651 \mathrm{~mm}$. The tiny slit width was 


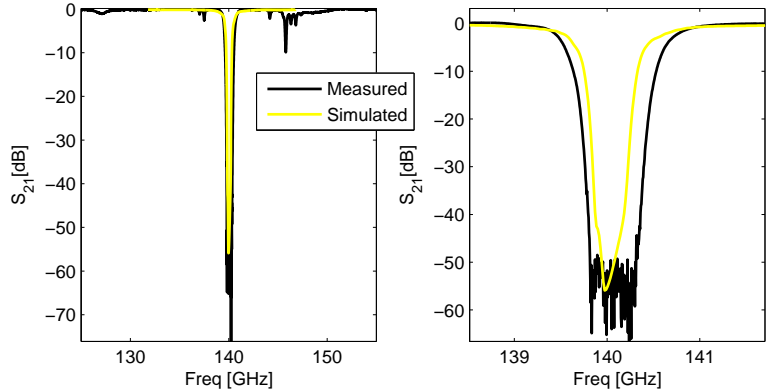

FIG. 9. Notch filter with ultra wide passband centered around $140 \mathrm{GHz}$

milled out in copper using a $0.1 \mathrm{~mm}$ diamond mill removing only $0.05 \mathrm{~mm}$ copper from the top in each run. After final adjustments using a network analyzer from Millimetre $\mathrm{AB}$, cavity lengths were spread from 4.92 to $5.6 \mathrm{~mm}$. Figure 9 compares the measured insertion loss with simulations. The typical loss in the passband is below $2 \mathrm{~dB}$. However, spurious notches appear at $\sim 145 \mathrm{GHz}$. The cavity lengths discrepancy of $5.6-4.92=0.68 \mathrm{~mm}$ can only explain $1 \mathrm{GHz}$ offset according to tab. I, which is not enough to explain the extra notches that show up. One likely explanation for these spurious notches is that the tuning screws leak a small portion of the electric field between the thread and the filter housing. The electric field inside the cavity therefore sees an electrically larger cavity than suggested in the simulated design. The notch filter performance can thus be improved by increasing the number of threads per axial distance or by increasing the cavity diameter, without changing the mode. However, the simulated value $(5.8 \mathrm{~mm})$ is close to the measured one $(\sim 5.4 \mathrm{~mm})$, showing good accuracy of the simulations. A way to avoid spurious notches is to improve the tuning screws using an alternative construction [8] or exciting the $\mathrm{TE}_{01}$ mode in the cavities. The current flow in this mode has only an azimuthal component, so the fields are not disturbed by poor electrical contact between the tuning screws and the cavity housing. $\mathrm{TE}_{01}$ is the third higher order mode with reduced passband coverage which is, however, still useful for CTS experiments and possibly for ECE. Another possible explanation to the spurious notches is an imperfect facing of the upper and bottom halves since both sides are milled individually.

The network analyzer dynamic range of $60 \mathrm{~dB}$ for $\mathrm{F}$ band designs will be the limiting factor in testing deep notches. A clear challenge in designing a deep notch filter is the limited network analyzer dynamic range. Therefore, systems that require minimum rejections of $60 \mathrm{~dB}$ should be designed as a cascade of two filters. However, the type of plasma experiment and constellation of the mm-wave receiver specify the requirements for the notch filter transmission and reflection parameters.

\section{CONCLUSIONS}

We have presented a notch filter with broad passband coverage. The T-junction slit coupling solution is suitable for rejection bandwidths of around $1 \mathrm{GHz}$ with broad passband coverage, typically more than $20 \mathrm{GHz}$. Simulations confirm that narrower rejection bandwidths are achievable by increasing the slit depth, which is relevant for CTS experiments. The key design parameters such as center frequency $f_{0}, 3 \mathrm{~dB}$ rejection bandwidth $\mathrm{BW}_{3 d B}$, and shape factor are estimated by tuning the geometry of the coupling slit and the cavity lengths. The sensitivity of measured insertion loss has been predicted with simulations by changing filter geometry. The simulation data in tab. I shows that small geometrical changes have considerable impact on filter parameters such as center frequency $f_{0}, 3 \mathrm{~dB}$ bandwidth $\mathrm{BW}_{3 d B}$, and shape factor.

\section{ACKNOWLEDGMENTS}

This work, supported by the European Communities under the contract of Association between EURATOM / Ris $\varnothing$ DTU, was partly carried out within the framework of the European Fusion Development Agreement. The views and opinions expressed herein do not necessarily reflect those of the European Commission. The authors are grateful to Ocleto D'Arcangelo, Giovanni Grossetti, and the rest of the team at IFP Milan for help with the measurements.
[1] F. Meo, H. Bindslev, S. Korsholm, F. Leipold, P. Michelsen, S. Nielsen, M. Salewski, P. Woskov, J. Leuterer, F. Stober, Wagner, and D. the ASDEX Upgrade team, Rev. Sci. Instrum., 79, 10E501 (2008).

[2] M. Salewski et al, Nuclear Fusion, 50, 035012 (2010).

[3] F. Meo et al, J. Phys.: Conf. Series, 227, 012010 (2010).

[4] S. Korsholm, H. Bindslev, F. Meo, F. Leipold, P. Michelsen, S. Michelsen, S. Nielsen, E. Tsakadze, P. Woskov, E. Westerhof, J. Oosterbeek, J. Hoekzema, F. Leuterer, and D. Wagner, Rev. Sci. Instrum., 77, 10E514 (2006).
[5] D. H. Wagner et al, IEEE Trans. Plasma Sci., 36, 324 (2008).

[6] D. H. Wagner et al, Nucl. Fusion, 48, 054006 (2008).

[7] Y. Dryagin, N. Scalyga, and T. Geist, Int. J. Infrared Millimeter Waves, 17, 1199 (1996).

[8] T. Geist and M. Bergbauer, Int. J. Infrared Millimeter Waves, 15, 2043 (1994).

[9] M. Salewski, F. Meo, H. Bindslev, V. Furtula, S. B. Korsholm, B. Lauritzen, F. Leipold, P. K. Michelsen, S. K. Nielsen, and E. Nonbøl, Rev. Sci. Instrum., 79, 10E729 (2008). 
[10] H. Bethe, M.I.T. Radiation Laboratory, Cambridge, Massachusetts, Report 43-22 (1943).

[11] P. A.Rizzi, Microwave Engineering - Passive Circuits (Prentice-Hall, Inc., 1988).
[12] Computer Simulation Design, Microwaves \& RF, www.cst.com. 